Pacific Journal of 


\title{
EXTENSION OF FLOWS VIA DISCONTINUOUS FUNCTIONS
}

\author{
P. D. Allenby and M. Sears
}

\begin{abstract}
We consider flows $(X, T)$ with $X$ compact Hausdorf, and suitable discontinuous functions $f: X \rightarrow W$ where $W$ is an arbitrary compact Hausdorff space. A ring extension of the ring of all continuous complex valued functions on $X(C(X))$ is formed and equipped with a norm. The Gelfand-Naimark theorem is applied to the completion of this normed ring to produce an almost one-to-one extension $\rho:\left(X_{f}, T\right) \rightarrow(X, T)$.

The question of isomorphism of flows $\left(X_{f}, T\right)$ and $\left(X_{g}, T\right)$ corresponding to functions $f$ and $g$ is discussed, as well as the lifting of dynamical properties from $(X, T)$ to $\left(X_{f}, T\right)$. Extension of flows via classes of discontinuous functions is considered, showing that no new examples arise in this way. A characterization theorem for extensions is proved when $T$ is locally compact Hausdorff, showing that every minimal almost one-to-one extension of $(X, T)$ can be obtained using our construction.
\end{abstract}

Introduction. In this paper we are concerned with creating extensions of flows by means of discontinuous functions. In essence the device is to add a suitable discontinuous function to a ring of continuous functions, obtaining a new structure space, in such a way that a new flow is generated which is an extension of the original one. Markley investigated extensions involving splitting along a single orbit by a somewhat different approach in [6].

The motivation for this theory is two fold. One hopes to modify existing examples to introduce new desired properties. We are able to introduce any compact metric space as a fibre in the extension in a similar way to that used by N. G. Markley in his situation. The classical Sturmian discrete flows involving adding two point fibres to a minimal circle rotation, thus obtaining highly proximal flows from equicontinuous ones, are probably the best known examples of this type of extension.

Another use for this extension process is to build models of the original flow. The initial flow is replaced by one of a desired type which is still close to the original in the sense that appropriate dynamical properties lift and the lifting map is "almost" an isomorphism i.e. it 
is injective on a dense second category set. This type of modelling has been discussed by several authors (see for example H. B. Keynes and J. R. Robertson [3], R. Bowen and P. Walters [1], H. B. Keynes and $M$. Sears [4]). Although a common philosophy underlies each of these situations, each is approached in a different way. We specify conditions under which a model of this type will arise automatically, giving machinery for this type of analysis.

Section 1 discusses the construction of the extension in detail. Sections 2,3 , and 4 are concerned with lifting properties, when two extensions will be isomorphic, and extensions using more than one discontinuous function. In $\S 5$ we note that for a locally compact acting group, our construction is completely general for minimal flows in that any minimal almost automorphic (almost one to one) extension arises in this way.

1. The construction. Let $(X, T)$ be a transformation group with compact Hausdorff phase space $X$, topological group $T$ acting freely on $X$. Suppose that $f: X \rightarrow W$ is a function from $X$ into another compact Hausdorff space $W$. Denote by $t \circ f$ the map of $X$ into $W$ defined by $t \circ f(x)=f(x t)$ for all $x \in X, t \in T$, and by $f_{x}$ the maps of $T$ into $W$ given by $f_{x}(t)=f(x t)$ for all $x \in X, t \in T$.

1.1. Definition. (i) $C(f)=\{x \in X: f$ is continuous at $x\}$

(ii) $C f=\{x \in X: x T \subset C(f)\}=\bigcap\{C(t \circ f): t \in T\}$

(iii) $\Omega(f, x)=\bigcap\{\operatorname{cl}(f(U \cap C f)): U \in \mathscr{U}(x)\}$, where $\mathscr{U}(x)$ is the neighbourhood filterbase at $x$, for all $x \in X$. We call $\Omega(f, x)$ the variation of $f$ at $x$.

When there is no danger of ambiguity, $C f$ is written as $C$.

Call $f$ acceptable if:

(a) $C$ is dense in $X$.

(b) $\left\{f_{x}: x \in C\right\}$ is an equicontinuous set of maps of $T$ into $W$.

(c) $f(x) \in \Omega(f, x)$ for all $x \in X$.

We will be concerned with the extension of $(X, T)$ by acceptable functions. We remark that any continuous function is acceptable.

Let $\mathscr{R}$ be the smallest ring containing $C(X)$ (the continuous complex-valued functions on $X$ ) and all maps of the form $g \circ$ $s \circ f$ where $g \in C(W)$ and $s \in T$, and identify functions $r, r^{\prime} \in \mathscr{R}$ if $r(c)=r^{\prime}(c)$ for all $c \in C$. If we define $\|\cdot\|: \mathscr{R} \rightarrow \mathbf{R}$ by $\|r\|=$ $\sup \{|r(x)|: x \in C\}$, then $(\mathscr{R},\|\cdot\|)$ is a normed ring. Denote its completion by $(\overline{\mathscr{R}},\|\cdot\|)$. If $c \in C$ and $\bar{r} \in \overline{\mathscr{R}}$, then $\bar{r}(c)$ means $\lim r_{n}(c)$ where $r_{n} \in \mathscr{R}$ for all $n$ and $r_{n} \rightarrow \bar{r}$. 


\subsection{Definition.}

$$
\Omega(r, x)=\bigcap\{\operatorname{cl}(r(U \cap C)): U \in \mathscr{U}(x)\} \quad \text { for all } r \in \overline{\mathscr{R}}, x \in X .
$$

By the Gelfand-Naimark theorem there is a compact Hausdorff space $\hat{X}$ and an isometric isomorphism $S$ of $\overline{\mathscr{R}}$ onto $C(\hat{X})$. We show that $(\hat{X}, T)$ is a transformation group (under a suitably defined action) which has $(X, T)$ as a factor. First we have

1.3. Theorem. If $X$ and $W$ are metrizable and $T$ is separable, then $\hat{X}$ is metrizable.

Proof. Since $C(X)$ and $C(W)$ are separable, we need only find a countable dense subset of $\{s \circ f: s \in T\}$ with topology given by the metric $d(s \circ f, t \circ f)=\sup \left\{d_{W}(f(x s), f(x t)): x \in C\right\}$. An obvious candidate is $\left\{s_{n} \circ f: n \in \mathbf{N}\right\}$ where $\left\{s_{n}: n \in \mathbf{N}\right\}$ is dense in $T$, and this is dense by the equicontinuity of $\left\{f_{x}: x \in X\right\}$.

The Action of $T$ on $\hat{X}$. Let $\hat{\rho}: C(X) \rightarrow C(\hat{X})$ be given by $\hat{\rho}=S \circ 1$ where 1 is the inclusion map of $C(X)$ into $\mathscr{R}$. Then $\hat{\rho}$ is an isometry of $C(X)$ into $C(\hat{X})$. Accordingly there is a continuous map $\rho: \hat{X} \rightarrow X$ with $\rho(\hat{X})$ dense in $X$ (so $\rho$ is onto $X$ ), and such that $\hat{\rho}(h)=h \circ \rho$ for all $h \in C(X)$.

Next, choose $s \in T$. Then $s$ determines an isometry $\hat{s}$ of $C(X)$ onto $C(X)$ given by $\hat{s}(g)=s \circ g$ for all $g \in C(X)$, where $s \circ g(x)=g(x s)$ for all $x \in X$. Similarly, $s$ determines an isometry of $\mathscr{R}$ onto $\mathscr{R}$, which can easily be seen to extend to all of $\overline{\mathscr{R}}$, giving an isometry $\bar{s}$ of $\overline{\mathscr{R}}$ onto $\overline{\mathscr{R}}$. We use the notation $\bar{s}(r)=s \circ r$ (with the usual meaning on $C)$ for all $r \in \overline{\mathscr{R}}$. Denote by $\hat{s}$ the corresponding isometry $S \bar{s} S^{-1}$ of $C(\hat{X})$ onto itself, and by $s$, again, the induced self-homeomorphism on $\hat{X}$. Writing $x s$ for $s(x)$, we have $\hat{s}(h)(x)=h(s(x))=s \circ h(x)$ for all $h \in C(\hat{X}), x \in \hat{X}$ and $s \in T$, and $\rho(x s)=\rho(x) s$ for all $x \in \hat{X}$ and $s \in T$.

If $s, t \in T$, clearly $x(s t)=(x s) t$ for all $x \in \hat{X}$, so we can consider $T$ to be a group acting freely on $\hat{X}$, and $\rho: \hat{X} \rightarrow X$ is equivariant. It remains to show that the map $\hat{X} \times T \rightarrow \hat{X}$ given by $(x, t) \rightarrow x t$ is continuous.

First note that since $\left\{f_{x}: x \in C\right\}$ is an equicontinuous family of maps, it follows that $\left\{r_{x}: x \in C\right\}$ is equicontinuous for all $r \in$ $\overline{\mathscr{R}}$, where $r_{x}(t)=r(x t)$. Also if $x \in C, s, t \in T$ and $r \in \overline{\mathscr{R}}$ then $\|s \circ r-t \circ r\|=\sup \left\{\left|r_{x}(s)-r_{x}(t)\right|: x \in C\right\}$. Hence if $\varepsilon>0$ and $t \in T$ there is an open neighbourhood $U(t)$ of $t$ such that $\|s \circ r-t \circ r\|<\varepsilon$ 
whenever $s \in U(t)$. Thus if $\left\{s_{\alpha}\right\}$ is a net in $T$ converging to $t$, then $s_{\alpha} \circ r \rightarrow t \circ r$ in $\overline{\mathscr{R}}$ for all $r \in \overline{\mathscr{R}}$. Now choose $g \in C(\hat{X})$. Then $g=S r$ for some $r \in \overline{\mathscr{R}}$, and $s_{\alpha} \circ g=\hat{s}_{\alpha}(g)=S \bar{s}_{\alpha} S^{-1}(g)=S\left(s_{\alpha} \circ r\right) \rightarrow$ $S(t \circ r)=S \bar{t} S^{-1}(g)=t \circ g$.

We have shown that if $\left\{s_{\alpha}\right\}$ is a net in $T$ and $s_{\alpha} \rightarrow t$, then $s_{\alpha} \circ g \rightarrow t \circ g$ for all $g \in C(\hat{X})$. We can now prove the continuity of the action $(x, t) \rightarrow x t$ of $T$ on $\hat{X}$.

Suppose that $\left\{\left(x_{\alpha}, s_{\alpha}\right)\right\}$ is a net in $\hat{X} \times T$ converging to $(x, t)$, and suppose wlog that $x_{\alpha} s_{\alpha} \rightarrow z$. We show that $z=x t$. Choose any $g \in C(\hat{X})$. Then $s_{\alpha} \circ g \rightarrow t \circ g$ i.e. $\left\|s_{\alpha} \circ g-t \circ g\right\| \rightarrow 0$ i.e.

$$
\sup \left\{\left|g\left(y s_{\alpha}\right)-g(y t)\right|: y \in \hat{X}\right\} \rightarrow 0 \text {. }
$$

Then $\left|g\left(x_{\alpha} s_{\alpha}\right)-g\left(x_{\alpha} t\right)\right| \rightarrow 0$ so $g(z)=g(x t)$. Since $g \in C(\hat{X})$ is arbitrary, $z=x t$. Hence $(\hat{X}, T)$ is a transformation group and $\rho$ : $(\hat{X}, T) \rightarrow(X, T)$ is a homomorphism.

Each $r \in \overline{\mathscr{R}}$ defines a map $r: C \rightarrow \mathbf{C}$ which is bounded and continuous. Hence we can define the map $\gamma: \overline{\mathscr{R}} \rightarrow C(\beta C)$ to be the map which takes $r \in \overline{\mathscr{R}}$ to its unique continuous extension $\gamma r: \beta C \rightarrow \mathbf{C}$, where $\beta C$ is the Stone-Čech compactification of $C$ (and we regard $C$ as a subset of $\beta C)$. $\gamma$ is clearly an isomorphism, so $\hat{h}=\gamma S^{-1}$ is an isomorphism of $C(\hat{X})$ into $C(\beta C)$. As usual there is an induced continuous onto map $h: \beta C \rightarrow \hat{X}$, say, such that $\hat{h}(g)=g \circ h$ for all $g \in C(\hat{X})$. Finally, let $\tau: \beta C \rightarrow X$ be the extension to $\beta C$ of the identity $1: C \rightarrow C$.

\subsection{THEOREM. (i) $\tau=\rho h$}

(ii) $(\hat{X}, h \mid C)$ is a compactification of $C$

(iii) $\rho: h(C) \rightarrow C$ is a homeomorphism of $h(C)$ onto $C$.

Proof. (i) If we let $\hat{\tau}(g)=g \circ \tau$ for all $g \in C(X)$, we obtain $\hat{\tau}=\hat{h} \hat{\rho}$.

(ii) Since $C$ is dense in $\beta C$ and $h$ is onto, $h(C)$ is dense in $\hat{X}$. If $c_{1}, c_{2} \in C$ and $h\left(c_{1}\right)=h\left(c_{2}\right)$, then $c_{1}=c_{2}$ by (i). Clearly $h: C \rightarrow h(C)$ is continuous, so we need only show that $h: C \rightarrow h(C)$ is closed, say.

First note that if $B \subset X$, then $B \cap C \subset C \subset \beta C$ and $h(B \cap C)=$ $\rho^{-1} B \cap h(C)$ from (i). Now, $\tau: C \rightarrow C$ is the identity homeomorphism of $C \subset \beta C$ onto $C \subset X$, so any closed subset of $C \subset \beta C$ has the form $B \cap C$ for some closed subset $B$ of $X$. But $h(B \cap C)=\rho^{-1} B \cap h(C)$ is closed in $h(C)$, so $h: C \rightarrow h(C)$ is a closed map.

(iii) This follows since every closed subset of $h(C)$ has the form $h(B \cap C)=\rho^{-1} B \cap C$ where $B$ ranges over the closed sets of $X$. 
Thus $\rho^{-1} C=h(C)$ is densely embedded as a copy of $C$ in $\hat{X}$, so we do not distinguish between $C \subset X$ and $C \subset \hat{X}$; we will usually write $\rho^{-1} c=c$ for $c \in C . \rho:(\hat{X}, T) \rightarrow(X, T)$ is thus an almost automorphic extension (i.e. there are fibres of $\rho$ which are points).

The lift of $f$ to $\hat{X}$. Let $t \in T$ and consider the map $(t \circ f)^{\wedge}: C(W) \rightarrow$ $\overline{\mathscr{R}}$ given by $(t \circ f) \wedge(g)=g \circ t \circ f$. Then $S(t \circ f)^{\wedge}$ is a homomorphism of $C(W)$ into $C(\hat{X})$, so there is a corresponding continuous map $(t \circ f)^{\prime}$ : $\hat{X} \rightarrow W$ such that $S(t \circ f)^{-}(g)=g \circ(t \circ f)^{\prime}$ for $g \in C(W)$. Let $f^{\prime}=(e \circ f)^{\prime}$ where $e$ is the identity of $T$. It follows that $(t \circ f)^{\prime}=t \circ f^{\prime}$ for all $t \in T$, so $S(t \circ f) \mathcal{Y}(g)=g \circ t \circ f^{\prime}$ for all $g \in C(W)$. The usefulness of these functions $t \circ f^{\prime}$ will be apparent after a short digression.

Suppose $\psi: \overline{\mathscr{R}} \rightarrow \mathbf{C}$ is a homomorphism of $\overline{\mathscr{R}}$ onto $\mathbf{C}$. Then $\psi S^{-1}$ is a homomorphism of $C(\hat{X})$ onto $\mathbf{C}$ and so there is a point $y \in \hat{X}$ such that $\psi S^{-1}=\theta_{y}$, where $\theta_{y}: C(\hat{X}) \rightarrow \mathbf{C}$ is defined by $\theta_{y}(g)=g(y)$ for all $g \in C(\hat{X})$. In particular if $x \in C$ and $\psi=\psi_{x}$, the evaluation homomorphism at $x$, then it can be shown that $\rho y=x$.

\subsection{LeMMA. If $r \in \overline{\mathscr{R}}$ and $c \in C$, then $(S r)(c)=r(c)$.}

Proof. By the above there is $y \in \rho^{-1} c$ such that $\psi_{c} S^{-1}=\theta_{y}$. But $\rho^{-1} c=c$, so $\psi_{c}=\theta_{c} S$. Now if $r \in \overline{\mathscr{R}}, r(c)=\psi_{c}(r)=\theta_{c}(S r)=$ $(S r)(c)$.

Denote the fibre $\rho^{-1} x$ over $x \in X$ by $\hat{X}_{x}$.

1.6. LeMMA. For $r \in \overline{\mathscr{R}}:$ (i) if $y \in \hat{X}$ then $(S r)(y) \in \Omega(r, \rho y)$ i.e. Sr $: \hat{X}_{x} \rightarrow \Omega(r, x)$ for all $x \in X$.

(ii) $S r: \hat{X}_{x} \rightarrow \Omega(r, x)$ is onto for all $x \in X$.

Proof. (i) Suppose that $y \in \hat{X}_{x}$. Choose a net $\left\{c_{\alpha}\right\} \subset C \subset \hat{X}$ such that $c_{\alpha} \rightarrow y$. Then $\operatorname{Sr}\left(c_{\alpha}\right)=r\left(c_{\alpha}\right)$ for all $\alpha$, and $\operatorname{Sr}\left(c_{\alpha}\right) \rightarrow \operatorname{Sr}(y)$. Thus $r\left(c_{\alpha}\right) \rightarrow \operatorname{Sr}(y)$. Finally, $c_{\alpha}=\rho c_{\alpha} \rightarrow \rho y=x$, so $\operatorname{Sr}(y) \in \Omega(r, x)$.

(ii) Let $a \in \Omega(r, x)$. Then there is a net $\left\{c_{\alpha}\right\} \subset C \subset X$ such that $c_{\alpha} \rightarrow x$ and $r\left(c_{\alpha}\right) \rightarrow a$. In $\hat{X}$ we may assume wlog that $c_{\alpha} \rightarrow y$, say. Clearly $\operatorname{Sr}(y)=a$ and $\rho y=x$.

We now return to the main development.

1.7. TheOREM. If $x \in X, t \in T$ and $g \in C(W)$ : (i) $S(g \circ t \circ f)=$ $g \circ t \circ f^{\prime}$.

(ii) $f^{\prime}(c)=f(c)$ for all $c \in C$. Moreover, $t \circ f^{\prime}(c)=t \circ f(c)$ for all $c \in C$. 
(iii) $t \circ f^{\prime}: \hat{X}_{x} \rightarrow \Omega(t \circ f, x)$ and is onto.

(iv) if $x \in C(t \circ f)$, then $f^{\prime}(y t)=f(x t)$ for all $y \in \hat{X}_{x}$

(v) $\Omega(f, x)=\{f(x)\}$ iff $x \in C(f)$.

Proof. (i) $S(g \circ t \circ f)=\left(S(t \circ f)^{\sim}\right)(g)=(t \circ f)^{\prime}(g)=g \circ t \circ f^{\prime}$.

(ii) For any $g \in C(W), g\left(f^{\prime}(c)\right)=S(g \circ e \circ f)(c)=g \circ e \circ f(c)=$ $g(f(c))$ by Lemma 1.5 and by (i) above. So $f^{\prime}(c)=f(c)$ for all $c \in C$.

(iii) This follows by (ii) and the fact that $C$ is dense in $X$ and $\hat{X}$.

(iv) follows from (iii).

(v) Suppose $x \notin C(f)$. Then there is an open neighbourhood $V$ of $f(x)$ in $W$ such that $f(U) \cap(W \backslash \bar{V}) \neq \varnothing$ for each $U \in \mathscr{U}(x)$, so for each $U \in \mathscr{U}(x)$ there is a point $x_{U} \in U$ with $f\left(x_{U}\right) \in W \backslash \bar{V}$. Since by hypothesis $f(y) \in \Omega(f, y)$ for each $y \in X$, it follows that for each $U \in \mathscr{U}(x)$ there is $c_{U} \in C$ such that $c_{U} \in U$ and $f\left(c_{U}\right) \in W \backslash \bar{V}$. Suppose wlog that $f\left(c_{U}\right) \rightarrow a$. Then $a \in \Omega(f, x)$ and $a \neq f(x)$, so $\Omega(f, x) \nsupseteq\{f(x)\}$. The converse is trivial.

1.8. COROLLARY. The following are equivalent:

(i) $\Omega(f, x t)=\{f(x t)\}$ for all $t \in T$.

(ii) $x \in C$.

(iii) $\hat{X}_{x}$ is a singleton.

So $\rho^{-1} x$ is a point if and only if $x \in C$.

An embedding of $\hat{X}$. For each $t \in T$, let $W_{t}=W$, and define $\mathscr{W}=\Pi\left\{W_{t}: t \in T\right\}$ and $F: \hat{X} \rightarrow \mathscr{W} \times X$ by $F=\Pi\left\{t \circ f^{\prime}: t \in T\right\} \times \rho$.

\subsection{TheOREM. $F$ is an embedding.}

Proof. $F$ is clearly continuous and closed, so we need only show that $F$ is injective. Suppose that $F\left(x_{1}\right)=F\left(x_{2}\right)$. Then $\rho x_{1}=\rho x_{2}=x$ say. We show that $(S r)\left(x_{1}\right)=(S r)\left(x_{2}\right)$ for all $r \in \overline{\mathscr{R}}$ i.e. $g\left(x_{1}\right)=g\left(x_{2}\right)$ for all $g \in C(\hat{X})$, whence $x_{1}=x_{2}$.

Clearly $(S r)\left(x_{1}\right)=(S r)\left(x_{2}\right)$ for all $r \in \mathscr{R}$, by (i) of Theorem 1.7, since $f^{\prime}\left(x_{1} t\right)=f^{\prime}\left(x_{2} t\right)$ for all $t \in T$ by hypothesis. Now let $r \in \overline{\mathscr{R}}$. Then there is a sequence $\left(r_{n}\right) \subset \mathscr{R}$ such that $\left\|r_{n}-r\right\| \rightarrow 0$, so that $\left\|S r_{n}-S r\right\| \rightarrow 0$. But

$$
\begin{aligned}
\left|\operatorname{Sr}\left(x_{1}\right)-\operatorname{Sr}\left(x_{2}\right)\right| \leq & \left|\operatorname{Sr}\left(x_{1}\right)-\operatorname{Sr}_{n}\left(x_{1}\right)\right|+\left|S r_{n}\left(x_{1}\right)-S r_{n}\left(x_{2}\right)\right| \\
& +\left|S r_{n}\left(x_{2}\right)-\operatorname{Sr}\left(x_{2}\right)\right| \\
& =\left|S r\left(x_{1}\right)-S r_{n}\left(x_{1}\right)\right|+\left|S r_{n}\left(x_{2}\right)-\operatorname{Sr}\left(x_{2}\right)\right| \text { for any } n .
\end{aligned}
$$

For $\varepsilon>0$ choose $n$ such that $\left\|S r_{n}-\operatorname{Sr}\right\|<\varepsilon / 2$. Then $\left|\operatorname{Sr}\left(x_{1}\right)-\operatorname{Sr}\left(x_{2}\right)\right|<$ $\varepsilon$. As $\varepsilon>0$ is arbitrary, $\operatorname{Sr}\left(x_{1}\right)=\operatorname{Sr}\left(x_{2}\right)$. 
1.10. Corollary. If $Y$ is a space and $G: Y \rightarrow \hat{X}$ is a map, then $G$ is continuous if and only if $\rho G$ is continuous and $t \circ f^{\prime} \circ G$ is continuous for all $t \in T$.

For all $x \in X$ let $D(x)=\{t \in T: x t \notin C(f)\}$, and let $\mathscr{D}(x)=$ $\Pi\{\Omega(f, x s): s \in D(x)\}$. Define $F_{x}: \hat{X}_{x} \rightarrow \mathscr{D}(x)$ by $F_{x}=\Pi\left\{s \circ f^{\prime}: s \in\right.$ $D(x)\}$.

1.11. Theorem. (i) If $D(x) \neq \varnothing$, then $F_{x}: \hat{X}_{x} \rightarrow \mathscr{D}(x)$ is an embedding.

(ii) $D(x)=\varnothing$ iff $x \in C$ iff $\hat{X}_{x}$ is a singleton.

Proof. (i) $F_{x}$ is continuous and closed. Suppose $F_{x}\left(x_{1}\right)=F_{x}\left(x_{2}\right)$ for some $x_{1}, x_{2} \in \hat{X}_{x}$. Then $t \circ f^{\prime}\left(x_{1}\right)=t \circ f^{\prime}\left(x_{2}\right)$ for all $t \in D(x)$, and the same is true of all $t \notin D(x)$ by Theorem 1.7 (iv), so that $F\left(x_{1}\right)=F\left(x_{2}\right)$. Now by $1.9, x_{1}=x_{2}$.

(ii) is clear by 1.8 .

An interesting special case of this theorem is

1.12. Corollary. Suppose that $D(x)$ is at most one point for all $x \in X$. If $x t \notin C(f)$, then $t \circ f^{\prime}: \hat{X}_{x} \rightarrow \Omega(f, x t)$ is a homeomorphism of $\hat{X}_{x}$ onto $\Omega(f, x t)$.

Proof. By 1.11 and 1.7(iii).

REMARKS. (i) It is not true in general that $F_{x}$ of 1.11 is onto $\mathscr{D}(x)$, as the examples below demonstrate.

(ii) Corollary 1.12 provides a way of "building in" precisely the fibres that we want. It is just necessary to define appropriate functions $f$ with a single discontinuity along any particular orbit.

1.13. Corollary. If $D(x)$ is at most one point for each $x \in X$, if each $\Omega(f, x)$ is connected, then for an open or closed $A \subset X, \rho^{-1} A$ is connected if and only if $A$ is connected. In particular, $\hat{X}$ is connected if $X$ is connected.

1.14. Examples. Let $X=[0,1)$ where addition in $X$ is modulo 1 i.e. $X$ is the circle group, and let $\phi: X \rightarrow X$ be a nontrivial group rotation. Then $X$ and $\phi$ determine a discrete transformation group 
$(X, \mathbf{Z})$. Let $I=[-1,1]$ and choose any $a, b \in I$. Choose $x \in X$ such that $0<x<\phi(x)<1$, and define $f: X \rightarrow I$ by

$$
f(t)= \begin{cases}a t / x, & 0 \leq t \leq x \\ \sin (1 /(t-x)), & x<t \leq \frac{1}{2}(x+\phi(x)) \\ \sin (1 /(\phi(x)-t)), & \frac{1}{2}(x+\phi(x)) \leq t<\phi(x) \\ (1-t) b /(1-\phi(x)), & \phi(x) \leq t<1\end{cases}
$$

Then $f$ is acceptable with

$$
\begin{gathered}
C(f)=X \backslash\{x, \phi(x)\}, \quad C f=X \backslash\left\{\phi^{n} x: n \in Z\right\}, \\
D(x)=\{0,1\} \quad \text { and } \quad \Omega(f, x)=I=\Omega(f, \phi x) .
\end{gathered}
$$

The extension $(\hat{X}, \mathbf{Z})$ of $(X, \mathbf{Z})$ by $f$ is determined by the space $\hat{X}$ and a surjective homeomorphism $\hat{\phi}: \hat{X} \rightarrow \hat{X}$ with $\rho \hat{\phi}=\phi \rho$.

If $F_{x}: \hat{X}_{x} \rightarrow \Omega(f, x) \times \Omega(f, \phi(x))$ is the embedding of Theorem 1.11 , then $F_{x}=f^{\prime} \times 1 \circ f^{\prime}=f^{\prime} \times f^{\prime} \hat{\phi}$, and it is easy to see that $F_{x}\left(\hat{X}_{x}\right)=\{a\} \times[-1,1] \cup[-1,1] \times\{b\}$, so that the fibre $\hat{X}_{x}$ over $x$ is isomorphic to this space. Clearly $F_{x}$ is not onto $\mathscr{D}(x)$.

Definition. The extension $(\hat{X}, T)$ of $(X, T)$ via an acceptable function $f$ is denoted by $\left(X_{f}, T\right)$ and is called the $f$-extension of $(X, T)$.

$A$ metric for $X_{f}$. We know that when $X$ and $W$ are metrizable and $T$ is separable, then $X_{f}$ is metrizable. The following corollary of 1.9 allows us to write down a convenient metric for $X_{f}$.

1.15. Theorem. Suppose $\left\{t_{n}: n \in \mathbf{N}\right\}$ is a dense subset of $T$, and let $F^{\prime}=\Pi\left\{t_{n} \circ f^{\prime}: n \in \mathbf{N}\right\} \times \rho$. If $W_{n}=W$ for all $n \in \mathbf{N}$, then $F^{\prime}: X_{f} \rightarrow \Pi\left\{W_{n}: n \in \mathbf{N}\right\} \times X$ is an embedding.

Now suppose $X$ and $W$ are metrizable with metrics $d_{X}$ and $d_{W}$ respectively. In view of 1.15 it is clear that the map $d_{f}: X_{f} \times X_{f} \rightarrow$ $[0, \infty)$ given by

$$
d_{f}(x, y)=\max \left[\sup _{n \in \mathbf{N}}\left\{\min \left(d_{W}\left(f^{\prime}\left(x t_{n}\right), f^{\prime}\left(y t_{n}\right)\right) / 2^{n}, 1\right)\right\}, d_{X}(\rho x, \rho y)\right]
$$

is a metric for $X_{f}$.

Another embedding of $X_{f}$. To end this section we state yet another corollary of 1.9. Let $W^{T}$ be the space of all continuous maps from $T$ into $W$ with compact-open topology. 
1.16. THEOREM. If $T$ is a locally compact Hausdorff group, then the map $x \rightarrow\left(f_{x}^{\prime}, \rho x\right)$ is an embedding of $X_{f}$ in $W^{T} \times X$.

Proof. Let $\pi: X_{f} \times T \rightarrow X_{f}$ be given by $\pi(x, t)=x t$, and let $G: X_{f} \times T \rightarrow W$ be given by $G=f^{\prime} \circ \pi$. Then $G$ is continuous, so the map $G^{\prime}: X_{f} \rightarrow W^{T}$ defined by $G^{\prime}(x)=f_{x}^{\prime}$ is also continuous. Finally, $G^{\prime} \times \rho: X_{f} \rightarrow W^{T} \times X$ is continuous, closed and, by 1.9 , injective.

\section{Lifting some dynamical properties.}

2.1. LEMMA. (i) If $x$ is a transitive point of $(X, T)$, then every point of $\rho^{-1} x$ is transitive in $\left(X_{f}, T\right)$.

(ii) If $\left(x, x^{\prime}\right)$ is a transitive point of $(X \times X, T)$, then every point of $\rho^{-1} x \times \rho^{-1} x^{\prime}$ is transitive in $\left(X_{f} \times X_{f}, T\right)$.

Proof. (i) Choose $y \in \rho^{-1} x$, and let $B=\overline{y T}$. Then $\rho B$ is closed in $X$ and $\rho B \supset x T$, so $\rho B=X$. Thus $B$ meets every fibre of $\rho$, so $B \supset C$. Therefore $B=X_{f}$.

(ii) Similar.

2.2. THEOREM. (i) $\left(X_{f}, T\right)$ is topologically transitive iff $(X, T)$ is topologically transitive.

(ii) $\left(X_{f}, T\right)$ is minimal iff $(X, T)$ is minimal.

(iii) $\left(X_{f}, T\right)$ is weak mixing iff $(X, T)$ is weak mixing.

2.3. THEOREM. If $(X, T)$ is minimal, then $\left(X_{f}, T\right)$ is a minimal proximal extension.

Proof. Suppose $y, y^{\prime} \in X_{f}$ are distinct and $\rho y=\rho y^{\prime}=x$. If $c \in C$ we can choose a net $\left(t_{\alpha}\right) \subset T$ such that $x t_{\alpha} \rightarrow c$. Hence $\rho\left(y t_{\alpha}\right) \rightarrow c$ and $\rho\left(y^{\prime} t_{\alpha}\right) \rightarrow c$, so $y t_{\alpha} \rightarrow c$ and $y^{\prime} t_{\alpha} \rightarrow c$.

This result is clearly true for any almost automorphic extension of a minimal flow (Brönstein 3.12.7).

2.4. Definition. (i) In the group $T$, write $t_{\alpha} \rightarrow \infty$ if the net $\left(t_{\alpha}\right) \subset$ $T$ has no convergent subnets. (If $T$ is locally compact Hausdorff, this means that $t_{\alpha} \rightarrow \infty$ in the one-point compactification $T^{*}=T \cup\{\infty\}$.)

(ii) If $(X, T)$ is a transformation group and $A \subset X$, then $A$ is called asymptotic if whenever $\left(t_{\alpha}\right) \subset T$ is a net with $t_{\alpha} \rightarrow \infty$ and there is $x \in A$ such that $x t_{\alpha} \rightarrow y$ say, then $z t_{\alpha} \rightarrow y$ for all $z \in A$.

(iii) An extension $\pi:(Y, T) \rightarrow(X, T)$ is called an asymptotic extension if $\pi^{-1} x$ is asymptotic for all $x \in X$. 
2.5. TheOREM. (i) If $x \in X$ and $\overline{D(x)}$ is compact in $T$, then $\rho^{-1} x$ is asymptotic.

(ii) If $\overline{D(x)}$ is compact for all $x \in X$, then $\rho:\left(X_{f}, T\right) \rightarrow(X, T)$ is an asymptotic extension.

Proof. (i) Suppose $x \in X$ with $\overline{D(x)}$ compact. Let $y \in \rho^{-1} x$ and let $\left(t_{\alpha}\right)$ be a net in $T$ such that $t_{\alpha} \rightarrow \infty$ and $y t_{\alpha} \rightarrow z$ say. Choose $y^{\prime} \in \rho^{-1} x$ and suppose wlog that $y^{\prime} t_{\alpha} \rightarrow z^{\prime}$. If $t \in T$ then $t_{\alpha} t \rightarrow \infty$, so there is $\beta$ such that $t_{\alpha} t \notin \overline{D(x)}$ for all $\alpha \geq \beta$, and since $\rho y^{\prime}=$ $\rho y=x, f^{\prime}\left(y^{\prime} t_{\alpha} t\right)=f\left(x t_{\alpha} t\right)=f^{\prime}\left(y t_{\alpha} t\right)$ for all $\alpha \geq \beta$, by 1.7. Therefore $f^{\prime}\left(z^{\prime} t\right)=f^{\prime}(z t)$. Since $t$ was arbitrary in $T, 1.9$ implies $z^{\prime}=z$.

(ii) is immediate, by (i).

2.6. CoRollary. If $D(x)$ is finite for all $x \in X, \rho:\left(X_{f}, T\right) \rightarrow(X, T)$ is an asymptotic extension.

As an application of the metric derived from 1.15 when $T=\mathbf{Z}$ and $\left(X, d_{X}\right)$ and $\left(W, d_{W}\right)$ are metric space, we examine expansiveness. An acceptable function $f: X \rightarrow W$ is said to satisfy property $(*)$ if there exists $\varepsilon>0$ such that whenever $\omega_{1}, \omega_{2} \in \Omega(f, x)$ for some $x \in X$ and $\omega_{1} \neq \omega_{2}$, then $d_{W}\left(\omega_{1}, \omega_{2}\right)>\varepsilon$.

2.7. THEOREM. If $f$ satisfies property $(*)$ on an expansive transformation group $(X, Z)$ with metric phase space, then $\left(X_{f}, \mathbf{Z}\right)$ is expansive.

Proof. Suppose $x_{1}, x_{2} \in X_{f}$ are distinct. If $\rho x_{1}=\rho x_{2}=x$ say, then by Theorems 1.9 and 1.7 (iii) there is an $n \in \mathbf{Z}$ such that $n \circ f^{\prime}\left(x_{1}\right) \neq n \circ$ $f^{\prime}\left(x_{2}\right)$ and both belong to $\Omega(f, x n)$. By hypothesis $d_{W}\left(f^{\prime}\left(x_{1} n\right), f^{\prime}\left(x_{2} n\right)\right)$ $>\varepsilon$. Now for any $m \in \mathbf{Z}$ we have

$$
d_{f}\left(x_{1} m, x_{2} m\right)=\sup \left\{\min \left(d_{W}\left(f^{\prime}\left(x_{1} i\right), f^{\prime}\left(x_{2} i\right)\right) / 2^{|i-m|}, 1\right): i \in \mathbf{Z}\right\}
$$

since $\rho x_{1}=\rho x_{2}$. Hence $d_{f}\left(x_{1} n, x_{2} n\right)>\min (\varepsilon, 1)$.

If on the other hand $\rho x_{1} \neq \rho x_{2}$, there is $n \in Z$ such that $d_{X}\left(\rho x_{1} n, \rho x_{2} n\right)>\delta$, where $\delta$ is an expansive constant for $(X, Z)$. Hence $\min (\varepsilon, \delta, 1)$ is an expansive constant for $\left(X_{f}, \mathbf{Z}\right)$.

REMARK. In the case where $D(x)$ is a singleton or empty for each $x \in X$, the expansiveness of $\left(X_{f}, \mathbf{Z}\right)$ implies that $f$ satisfies property $(*)$. 
2.8. Proposition. Let $f: X \rightarrow W$ be acceptable on $(X, T)$, and suppose that $T$ is separable with $\left\{t_{n}: n \in \mathbf{N}\right\}$ dense in $T$. Then

(i) $C=\bigcap\left\{C\left(t_{n} \circ f\right): n \in \mathbf{N}\right\}$.

(ii) If $C\left(t_{n} \circ f\right)$ is a $G_{\delta}$ set for each $n \in \mathbf{N}$, then $C \subset X$ and $\rho^{-1} C \subset X_{f}$ are $G_{\delta}$ sets, so $C$ and $\rho^{-1} C$ are dense second category sets in their respective spaces.

Proof. (i) By definition $C \subset \bigcap\left\{C\left(t_{n} \circ f\right): n \in \mathbf{N}\right\}$. Now suppose $x \in \bigcap\left\{C\left(t_{n} \circ f\right): n \in \mathbf{N}\right\}$, and let $y, y^{\prime} \in \rho^{-1} x$. If $t \in T$ is arbitrary, there is a sequence $\left(t_{k}\right)$ from $\left\{t_{n}: n \in \mathbf{N}\right\}$ such that $t_{k} \rightarrow t$. Now $f^{\prime}\left(y t_{k}\right)=f^{\prime}\left(y^{\prime} t_{k}\right)$ for all $k$ by $1.7(\mathrm{iv})$, so $f^{\prime}(y t)=f^{\prime}\left(y^{\prime} t\right)$ by continuity. 1.9 now implies that $y=y^{\prime}$, so $\rho^{-1} x$ is a singleton. Now by $1.11(\mathrm{ii})$, $x \in C$.

(ii) follows from (i).

The results of this section enable us to construct models of flows in the sense described in the introduction. All that is required (provided $T$ is separable) is that $C\left(t_{n} \circ f\right)$ is a dense $G_{\delta}$ set for each $t_{n}$ (usually we can arrange that these sets are actually open). Dynamical properties lift as in the above theorems.

In the case of discrete flows, the process is immediate by choosing a suitable function (e.g. a characteristic function defined on the circle with minimal rotation immediately produces a minimal extension and the lift is injective except on a set of $1^{\circ}$ Category). When handling continuous flows we need to construct our functions more carefully using sections. As an example we outline the construction of a suspension model for real flows using our approach (see [4]).

Definition. Let $(X, \mathbf{R})$ be a real flow. A section of a point $x \in X$ is a closed set $S \subset X$ with $x \in S$ and such that for some $\delta>0$ (a section time), $S \cap S(0, \delta]=S \cap S[-\delta, 0)=\varnothing$.

We define $S^{*}$ as the relative interior of $S$ i.e. $S^{*}=\operatorname{Int}(S(-\delta, \delta)) \cap$ $S$. Then $S^{*}$ is open in $S, S^{*}(-\delta, \delta)$ is open in $X$, and we can choose sections in such a way that $S^{*}$ is dense in $S$. Furthermore we have the following result (Lemma 7 of [1]):

2.9. TheOREM. There is $a \zeta>0$ so that the following holds: For each $\alpha>0$ there is a finite family $\mathscr{S}$ of pairwise disjoint sections of time $\zeta$ and diameter at most $\alpha$ such that $X=Y[-\alpha, 0]=Y[0, \alpha]$ where $Y=\bigcup_{S \in \mathscr{S}} S^{*}$.

2.10. THEOREM. Every real flow is modelled by a real suspension. 
Proof. We will construct an extension which has a global section i.e. a section $\Gamma$ with the property that every orbit intersects $\Gamma$ and that $\Gamma(-\delta, \delta)$ is open for $\delta$ sufficiently small. It is well known that such a flow can be realised as a suspension over $\Gamma$.

Let $\mathscr{S}=\left\{S_{1}, S_{2}, \ldots, S_{n}\right\}$ be chosen as above with $\zeta$ small enough so that $S_{i}[-\zeta, \zeta] \cap S_{j}[-\zeta, \zeta]=\varnothing$ for $i \neq j$. Define $f$ on $X$ by $f(x \cdot t)=$ $1-|t| / \zeta$ if $x \in S_{i}$ (for some $i$ ) and $|t|<\zeta$, and $f(x)=0$ otherwise. It is clear that $f$ is an acceptable function which satisfies the conditions of Proposition 2.8. Form the extension $\left(X_{f}, \mathbf{R}\right)$. We will show that $\Gamma=f^{\prime-1}(1)$ is a global section for this flow. First note that $\Gamma$ will be a section with time $\zeta$.

Now if $x \in X_{f}$, then for some $t \in \mathbf{R}$ and $S_{i}, \rho(x t) \in S_{i}^{*}$. Since $S_{i}^{*} \subset$ $C(f), f^{\prime}(x t)=f(\rho(x) t)=1$. Thus every orbit intersects $\Gamma$. Suppose that $\Gamma(-\zeta, \zeta)$ is not open. Then we can find a point $x \in \Gamma(-\zeta, \zeta)$ and a sequence $c_{n} \in \rho^{-1}(C)$ with $c_{n} \notin \Gamma(-\zeta, \zeta)$ and $c_{n} \rightarrow x$. Equivalently, we can find $x \in \Gamma$ and a sequence $c_{n} \notin \Gamma(-\delta, \delta)$ and $c_{n} \rightarrow x$ for some $\delta>0$. Now $f\left(c_{n}\right)=f^{\prime}\left(c_{n}\right) \rightarrow 1$. We deduce $c_{n}=s_{n} t_{n}$ where $c_{n} \in S_{i}$ for some $i$ and $t_{n} \in \mathbf{R}$ with $t_{n} \rightarrow 0$. Thus $c_{n}\left(-t_{n}\right) \in S_{i}$, so $f^{\prime}\left(c_{n}\left(-t_{n}\right)\right)=1$ and for $n$ sufficiently large $c_{n} \in \Gamma(-\delta, \delta)$ which is a contradiction.

REMARK. Since $\mathbf{R}^{n}$ parallels can be obtained for Theorem 2.9, the same process can be used to obtain models for $R^{n}$ flows which have global sections. A difficult open question is which of those flows are $\mathbf{R}^{n}$ suspensions (see [5]).

3. An isomorphism theorem. Let $f$ and $g$ be acceptable functions on a flow $(X, T)$ producing extensions $\rho_{f}:\left(X_{f}, T\right) \rightarrow(X, T)$ and $\rho_{g}:\left(X_{g}, T\right) \rightarrow(X, T)$ respectively. For each $x \in X$ let $\omega_{f}(x)=$ $\Pi\{\Omega(f, x t): t \in T\} \times\{x\}$, and let $\Omega(f)=\bigcup\left\{\omega_{f}(x): x \in X\right\}$. Referring to Theorem 1.9 and its notation, regard $\Omega(f)$ as a subspace of $\mathscr{W}_{f} \times X$; then $F: X_{f} \rightarrow \Omega(f)$ is an embedding. Let $G: X_{g} \rightarrow \Omega(g)$ be the corresponding embedding for $X_{g}$.

One might imagine that if $f$ and $g$ have homeomorphic variations at each point, the resulting extensions $\left(X_{f}, T\right)$ and $\left(X_{g}, T\right)$ would be isomorphic. In fact, one needs some sort of uniformity across the variations in the sense that not only can we map $\omega_{f}(x)$ homeomorphically onto $\omega_{g}(x)$, but that the resulting collection of homeomorphisms acts in a continuous way from $\Omega(f)$ to $\Omega(g)$. In this case the resulting isomorphism maps fibres to fibres. This is made concrete in the next theorem. 
3.1. TheOREM. Suppose that $f$ and $g$ are acceptable on $(X, T)$ and let $m:(X, T) \rightarrow(X, T)$ be a homomorphism. If there is a continuous surjection $m^{\prime}: \Omega(f) \rightarrow \Omega(g)$ such that $m^{\prime}: \omega_{f}(x) \rightarrow \omega_{g}(m x)$ and is onto for all $x \in X$, then there is a homomorphism $\pi:\left(X_{f}, T\right) \rightarrow\left(X_{g}, T\right)$ such that $\rho_{g} \pi=m \rho_{f}$. Moreover, $\pi$ is an isomorphism if $m^{\prime}$ is injective.

Proof. If $c \in C f$ then $\omega_{f}(c)$ is a point. Therefore $\omega_{g}(m c)$ is a point, so $m c \in C g$. Thus $m(C f) \subset C g$. Also, $m^{\prime}\left(\left\{f^{\prime}(c t)\right\}_{t} \times\{c\}\right)=$ $\left(\left\{g^{\prime}(m c t)\right\}_{t} \times\{m c\}\right)$ so $m^{\prime} F=G m$ on $C f$.

If $x \in X_{f}$, choose a net $\left(c_{\alpha}\right) \subset C f \subset X_{f}$ converging to $x$. We may assume that $m c_{\alpha} \rightarrow y$ say, in $X_{g}$. But $m^{\prime} F\left(c_{\alpha}\right) \rightarrow m^{\prime} F(x)$ and $G m\left(c_{\alpha}\right) \rightarrow G(y)$, so $m^{\prime} F(x)=G(y)$. This shows that $m^{\prime} F\left(X_{f}\right) \subset$ $G\left(X_{g}\right)$, so $\pi: X_{f} \rightarrow X_{g}$ given by $\pi=G^{-1} m^{\prime} F$ is well-defined and continuous.

$\pi$ is equivariant. For if $c \in C f$ and $t \in T$, then $\pi(c t)=m(c t)=$ $m(c) t=\pi(c) t$ and continuity does the rest. Similarly, if $c \in C f$ then $\rho_{g} \pi(c)=\rho_{g} m(c)=m(c)=m \rho_{f}(c)$, so again by continuity, $\rho_{g} \pi=m \rho_{f}$.

$\pi$ is also surjective: $\rho_{g} \pi\left(X_{f}\right)=m \rho_{f}\left(X_{f}\right)=m X=X$ as $m$ is surjective, so $\pi\left(X_{f}\right)$ meets every fibre of $X_{g}$. Thus $\pi\left(X_{f}\right) \supset C g$, and, being closed, $\pi\left(X_{f}\right)=X_{g}$. Clearly $\pi$ is injective if $m^{\prime}$ is injective.

3.2. Corollary. Let $f: X \rightarrow W$ be acceptable on $(X, T)$ and let $W^{\prime}$ be a compact Hausdorff space and $H: W \rightarrow W^{\prime}$ continuous. Then $H f: X \rightarrow W^{\prime}$ is acceptable and there is a homomorphism $\pi:\left(X_{f}, T\right) \rightarrow$ $\left(X_{H f}, T\right)$ such that $\rho_{H f} \pi=\rho_{f} . \pi$ is injective if $H$ is injective.

3.3. Corollary. If $f: X \rightarrow W$ is acceptable on $(X, T)$, then there is an acceptable map $g: X \rightarrow W \times X$ such that $\Omega(g, x) \cap \Omega(g, y)=\varnothing$ for all distinct $x, y \in X$, and an isomorphism $\pi:\left(X_{g}, T\right) \rightarrow\left(X_{f}, T\right)$ such that $\rho_{f} \pi=\rho_{g}$. 3.1 .

Proof. Let $g: X \rightarrow W \times X$ be defined by $g(x)=(f(x), x)$ and apply

In view of 3.3, we remark that any acceptable map $f$ on a transformation group $(X, T)$ can be replaced by an injective acceptable map producing the same extension of $(X, T)$.

4. Class extensions. So far we have considered the extension of flows by means of a single function. A natural question is whether we could obtain different examples by using classes of functions. We will 
show below that this is not the case. We just sketch the development which parallels $\S 1$.

Let $\mathscr{F}=\left\{f_{i}: i \in \Lambda\right\}$ be a set of functions on the phase space $X$ of a transformation group $(X, T)$, the range space $W_{i}$ of $f_{i}$ being compact Hausdorff for each $i \in \Lambda$. For each $i$ let $C_{i}=C f_{i}$, and let $f_{i x}$ $(x \in X), t \circ f_{i}(t \in T)$ and $\Omega\left(f_{i}, x\right)$ (wrt $C_{i}$ ) have their usual meanings.

4.1. Definition. $\mathscr{F}$ is called acceptable on $(X, T)$ if:

(i) $C=\bigcap\left\{C_{i}: i \in \Lambda\right\}$ is dense in $X$.

(ii) $\left\{f_{i x}: x \in C_{i}\right\}$ is equicontinuous on $T$ for each $i \in \Lambda$.

(iii) $f_{i}(x) \in \Omega\left(f_{i}, x\right)$ for each $x \in X, i \in \Lambda$.

(iv) $\left\{f_{i x}: i \in \Lambda, x \in C\right\}$ is equicontinuous.

Define $\mathscr{R} \mathscr{F}$ to be the smallest ring containing $C(X)$ and all compositions $g \circ t \circ f_{i}$ where $t \in T, i \in \Lambda$ and $g \in C\left(W_{i}\right)$. Proceeding in a way similar to that of $\S 1$, one obtains a transformation group $(X \mathscr{F}, T)$ with associated homomorphism $\rho:(X \mathscr{F}, T) \rightarrow(X, T)$, and the set $C$ is densely embedded in $X \mathscr{F}$ with $\rho^{-1}(x)$ a singleton iff $x \in C$.

If we let $f: X \rightarrow \Pi\left\{W_{i}: i \in \Lambda\right\}$ be defined by $f=\Pi f_{i}$, then $f$ is acceptable on $(X, T)$ and

4.2. TheOREM. There is an isomorphism $\pi:\left(X_{f}, T\right) \rightarrow(X \mathscr{F}, T)$ such that $\rho \pi=\rho_{f}$.

On the other hand, construct the extension $\rho_{i}:\left(X_{i}, T\right) \rightarrow(X, T)$ of $(X, T)$ via $f_{i}$ for each $i \in \Lambda$. Let $C^{\prime} \subset \Pi\left\{X_{i}: i \in \Lambda\right\}$ be defined by $C^{\prime}=\left\{\left\{c_{i}\right\}: c_{i}=c \forall i\right.$ for some $\left.c \in C\right\}$. Let $Y=\operatorname{cl}(C)$ in $\Pi\left\{X_{i}: i \in \Lambda\right\}$. Then $(Y, T)$ is a transformation group and if $\left\{y_{i}\right\} \in Y, \rho_{i} y_{i}=\rho_{j} y_{j}$ for each $i, j \in \Lambda$. Let $p:(Y, T) \rightarrow(X, T)$ be any of the maps $\rho_{i}$.

4.3. THEOREM. There is an isomorphism $\pi:(X \mathscr{F}, T) \rightarrow(Y, T)$ such that $p \pi=\rho$.

4.4. THEOREM. Let $(Z, T)$ be a transformation group and $\mathscr{F}$ an acceptable set of functions on $(X, T)$, and suppose that for each $i \in$ $\Lambda$ there is a homomorphism $\pi_{i}:(Z, T) \rightarrow\left(X_{i}, T\right)$ such that $\rho_{i} \pi_{i}=$ $\rho_{j} \pi_{j}$ for each $i, j \in \Lambda$. Then there is a homomorphism $\pi:(Z, T) \rightarrow$ $(X \mathscr{F}, T)$. If $\left\{\pi_{i}: i \in \Lambda\right\}$ separates points of $Z, \pi$ is an isomorphism.

4.4. Definition. The fibered product \#\{(X, $T): i \in \Lambda\}$ of a set of extensions $p_{i}:\left(X_{i}, T\right) \rightarrow(X, T)$ is the transformation group $\left(\# X_{i}, T\right)$ where

$$
\# X_{i}=\left\{\left\{x_{i}\right\} \in \Pi X_{i}: p_{i} x_{i}=p_{j} x_{j} \forall i, j \in \Lambda\right\}
$$


It is clear that with notation as above, $(Y, T)$ is a sub-transformation group of $\#\left(X_{i}, T\right)$. In the case where $C_{i} \cup C_{j}=X$ whenever $i, j \in \Lambda$ are distinct, it is easy to show that $Y=\# X_{i}$.

4.5. Corollary (of 4.3). If $C_{i} \cup C_{j}=X$ for all distinct $i, j \in \Lambda$, then there is an isomorphism $\pi:(X \mathscr{F}, T) \rightarrow \#\left(X_{i}, T\right)$ such that $p \pi=\rho$.

4.6. THEOREM. Let $(X, T)$ be minimal and let $f, g$ be acceptable functions on $(X, T)$ such that $C f \cap C g$ is non-empty and $C f \cup C g=X$. Then the extensions $\rho_{f}$ and $\rho_{g}$ are disjoint.

Proof. By the preceding results, $\left(X_{f}, T\right) \#\left(X_{g}, T\right)$ is the $\{f, g\}$ extension of $(X, T)$, so is minimal. The result follows as in [2].

5. Characterization of $f$-extensions. The characterization of $f$-extensions presented here shows that every minimal almost automorphic extension of a transformation group $(X, T)$, with compact Hausdorff phase space and locally compact Hausdorff group $T$, is an $f$-extension of $(X, T)$. This gives an alternative proof of Theorem 4.2 in this situation.

5.1. LemMA. Let $(X, T)$ be a transformation group with $T$ locally compact Hausdorff. Consider $X$ as a set of maps of $T$ into $X$. Then $X$ is equicontinuous on $T$.

Proof. $\pi: X \times T \rightarrow X$ given by $\pi(x, t)=x t$ is continuous, so $\hat{\pi}: X \rightarrow X^{T}$ is continuous and injective, where $\hat{\pi}(x)(t)=\pi(x, t)$, and where $X^{T}$ is the set of continuous maps from $T$ into $X$ with compactopen topology. As $X^{T}$ is Hausdorff and $X$ is compact, $\hat{\pi}$ is a closed map, so $\hat{\pi} X$ is homeomorphic to $X$. But $\hat{\pi} X \subset X^{T}$ is closed and compact, so is equicontinuous.

5.2. TheOREM. Let $\pi:(Y, T) \rightarrow(X, T)$ be a homomorphism, where $T$ is locally compact and Hausdorff. Suppose that the set of all singleton fibres of $\pi$ is dense in $Y$. Then there is an acceptable $f: X \rightarrow Y$ such that $f^{\prime}:\left(X_{f}, T\right) \rightarrow(Y, T)$ is an isomorphism and $\pi f^{\prime}=\rho_{f}$.

This immediately shows that every minimal almost automorphic extension is an $f$-extension:

5.3. Corollary. If $\pi:(Y, T) \rightarrow(X, T)$ is a minimal almost automorphic extension and $T$ is locally compact Hausdorff, then there is an 
acceptable $f: X \rightarrow Y$ such that $f^{\prime}:\left(X_{f}, T\right) \rightarrow(Y, T)$ is an isomorphism and $\pi f^{\prime}=\rho_{f}$.

Proof of 5.2. Define $C^{\prime}=\left\{y: \pi^{-1} \pi(y)=\{y\}, y \in Y\right\}$. Then $C^{\prime}$ is dense in $Y$ and $C=\pi C^{\prime}$ is dense in $X$. Next we define $f: X \rightarrow Y$ as follows. If $c \in C$, let $f(c)=\pi^{-1} c$. On the other hand, let $O$ be any orbit in $X$ with $O \cap C=\varnothing$. Choose $x_{0} \in O$ and $f\left(x_{0}\right) \in \pi^{-1} x_{0}$, and define $f\left(x_{0} t\right)=f\left(x_{0}\right) t$ for all $t \in T$. So $f: X \rightarrow Y$ is defined (using the axiom of choice).

(i) $C(f) \subset C$. Let $x \in C(f)$ and suppose $y, y^{\prime} \in \pi^{-1} x$. As $C^{\prime}$ is dense in $Y$, suppose $c_{\alpha} \rightarrow y, c_{\alpha}^{\prime} \rightarrow y^{\prime}$ where $\left(c_{\alpha}\right)$ and $\left(c_{\alpha}^{\prime}\right)$ are nets in $C^{\prime}$. Then $\pi c_{\alpha} \rightarrow x$ and $\pi c_{\alpha}^{\prime} \rightarrow x$. Now as $x \in C(f), f\left(\pi c_{\alpha}\right) \rightarrow f(x)$ and $f\left(\pi c_{\alpha}^{\prime}\right) \rightarrow f(x)$. But $f(\pi c)=c$ for all $c \in C$, so $c_{\alpha} \rightarrow f(x)$ and $c_{\alpha}^{\prime} \rightarrow f(x)$ i.e. $y=y^{\prime}$. Thus $\pi^{-1} x$ is a singleton, so $x \in C$.

(ii) $C \subset C(f)$. Suppose $c \in C$. Choose a net $x_{\alpha} \rightarrow c$ in $X$ such that $f\left(x_{\alpha}\right) \rightarrow l$. As $\pi f\left(x_{\alpha}\right)=x_{\alpha}, \pi(l)=c$. Hence $l=f(c)$ as $l \in C^{\prime}$.

Hence $C(f)=C$, so $C f=C$.

(iii) $\Omega(f, x)=\pi^{-1} x$ for all $x \in X$. If $y \in \Omega(f, x)$, there is a net $c_{\alpha} \rightarrow x$ from $C$ such that $f\left(c_{\alpha}\right) \rightarrow y$. Thus $\pi f\left(c_{\alpha}\right) \rightarrow \pi y$, i.e., $c_{\alpha} \rightarrow \pi y$. Thus $\pi y=x$, i.e., $y \in \pi^{-1} x$. On the other hand, let $y \in \pi^{-1} x$. Then there is a net $\left(c_{\alpha}^{\prime}\right) \subset C^{\prime}$ such that $c_{\alpha}^{\prime} \rightarrow y$, i.e., $f\left(\pi c_{\alpha}^{\prime}\right) \rightarrow y$. But $\pi c_{\alpha}^{\prime} \rightarrow \pi y=x$, so $y \in \Omega(f, x)$.

Hence $f(x) \in \Omega(f, x)$ for all $x \in X$.

(iv) $\left\{f_{x}: x \in C\right\}$ is equicontinuous on $T$. This follows by Lemma 5.1 applied to $(Y, T)$.

Hence $f: X \rightarrow Y$ is acceptable on $(X, T)$, so we can construct the $f$-extension $\rho:\left(X_{f}, T\right) \rightarrow(X, T)$. If $f^{\prime}$ is the usual lift of $f$ to $X_{f}$, then $f^{\prime}: X_{f} \rightarrow Y$ is onto as $f^{\prime}\left(X_{f}\right) \supset f^{\prime}(C)=f(C)=C^{\prime}$ and $f^{\prime}$ is closed. $f^{\prime}$ is also clearly equivariant, and since $\pi f^{\prime}(c)=\pi f(c)=c=\rho c$ $\forall c \in C ; \pi f^{\prime}=\rho$. Lastly, we show that $f^{\prime}$ is injective.

Suppose $x, x^{\prime} \in X_{f}$ and $f^{\prime}(x)=f^{\prime}\left(x^{\prime}\right)$. Then $\rho x=\pi f^{\prime}(x)=$ $\pi f^{\prime}\left(x^{\prime}\right)=\rho x^{\prime}$. Now the map $F$ of Theorem 1.9 is an embedding. But for each $t \in T, f^{\prime}(x t)=f^{\prime}(x) t=f^{\prime}\left(x^{\prime}\right) t=f^{\prime}\left(x^{\prime} t\right)$, so $F(x)=F\left(x^{\prime}\right)$. Hence $x=x^{\prime}$.

Thus $f^{\prime}:\left(X_{f} T\right) \rightarrow(Y, T)$ is an isomorphism and $\pi f^{\prime}=\rho$.

5.4. Corollary. Suppose $(X, T)$ and $(Y, T)$ are transformation groups with $T$ locally compact Hausdorff, and let $\left(X_{f}, T\right)$ be an $f$ extension of $(X, T)$. If there are homomorphisms $p:\left(X_{f} T\right) \rightarrow(Y, T)$ 
and $\pi:(Y, T) \rightarrow(X, T)$ such that $\pi p=\rho_{f}$, then there is an acceptable $g: X \rightarrow Y$ and an isomorphism $G:(Y, T) \rightarrow\left(X_{g}, T\right)$ such that $\rho_{g} G=\pi$.

Proof. We need only show that $A=\left\{y: y \in Y\right.$ and $\left.\pi^{-1} \pi y=\{y\}\right\}$ is dense in $Y$. But $A \supset p C f$ (where $C f \subset X_{f}$ ).

\section{REFERENCES}

[1] R. Bowen and P. Walters, Expansive one parameter flows, J. Differential Equations, 12 (1972), 180-193.

[2] I. U. Brönstein, Extensions of Minimal Transformation Groups, Sijthoff and Noordhoff, 1979.

[3] H. B. Keynes and J. Robertson, Generators for topological entropy and expansiveness, Math. Systems Theory, 3 (1969), 51-59.

[4] H. B. Keynes and M. Sears, Modelling expansion in real flows, Pacific J. Math., 85 (1979), 111-124.

[5] , Time changes for $\mathbf{R}^{n}$ flows and suspensions, Pacific J. Math., 130 (1987), 97-113.

[6] N. G. Markley, F-minimal sets, Trans. Amer. Math. Soc., 163 (1972), 85-100.

Received March 20, 1987.

UNIVERSITY OF THE WITWATERSRAND

JohANNESBURG, South Africa 



\section{EDITORS}

\author{
V. S. VARADARAJAN \\ (Managing Editor) \\ University of California \\ Los Angeles, CA 90024 \\ HeRBert Clemens \\ University of Utah \\ Salt Lake City, UT 84112 \\ R. FINN \\ Stanford University \\ Stanford, CA 94305
}

\author{
HERMANN FLASCHKA \\ University of Arizona \\ Tucson, AZ 85721
}

RAMESh A. GANGOLLI University of Washington Seattle, WA 98195

VAUGHAN F. R. JONES University of California

Berkeley, CA 94720
ROBION KIRBY

University of California

Berkeley, CA 94720

C. C. MOORE

University of California

Berkeley, CA 94720

HAROLD STARK

University of California, San Diego

La Jolla, CA 92093

\section{ASSOCIATE EDITORS}
R. ARENS
E. F. BECKENBACH
B. H. NEUMANN
F. WOLF
K. YOSHIDA

(1906-1982)

\section{SUPPORTING INSTITUTIONS}

UNIVERSITY OF ARIZONA
UNIVERSITY OF BRITISH COLUMBIA
CALIFORNIA INSTITUTE OF TECHNOLOGY
UNIVERSITY OF CALIFORNIA
MONTANA STATE UNIVERSITY
UNIVERSITY OF NEVADA, RENO
NEW MEXICO STATE UNIVERSITY
OREGON STATE UNIVERSITY

UNIVERSITY OF ARIZONA

UNIVERSITY OF BRITISH COLUMBIA

UNIVERSITY OF CALIFORNIA

MONTANA STATE UNIVERSITY

NEW MEXICO STATE UNIVERSITY

OREGON STATE UNIVERSITY

\author{
UNIVERSITY OF OREGON \\ UNIVERSITY OF SOUTHERN CALIFORNIA \\ STANFORD UNIVERSITY \\ UNIVERSITY OF HAWAII \\ UNIVERSITY OF TOKYO \\ UNIVERSITY OF UTAH \\ WASHINGTON STATE UNIVERSITY \\ UNIVERSITY OF WASHINGTON
}

The Supporting Institutions listed above contribute to the cost of publication of this Journal, but they are not owners or publishers and have no responsibility for its content or policies.

Mathematical papers intended for publication in the Pacific Journal of Mathematics should be in typed form or offset-reproduced (not dittoed), double spaced with large margins. Please do not use built up fractions in the text of the manuscript. However, you may use them in the displayed equations. Underline Greek letters in red, German in green, and script in blue. The first paragraph must be capable of being used separately as a synopsis of the entire paper. In particular it should contain no bibliographic references. Please propose a heading for the odd numbered pages of less than 35 characters. Manuscripts, in triplicate, may be sent to any one of the editors. Please classify according to the scheme of Math. Reviews, Index to Vol. 39. Supply name and address of author to whom proofs should be sent. All other communications should be addressed to the managing editor, or Elaine Barth, University of California, Los Angeles, California 90024.

There are page-charges associated with articles appearing in the Pacific Journal of Mathematics. These charges are expected to be paid by the author's University, Government Agency or Company. If the author or authors do not have access to such Institutional support these charges are waived. Single authors will receive 50 free reprints; joint authors will receive a total of 100 free reprints. Additional copies may be obtained at cost in multiples of 50 .

The Pacific Journal of Mathematics is issued monthly as of January 1966. Regular subscription rate: $\$ 190.00$ a year (5 Vols., 10 issues). Special rate: $\$ 95.00$ a year to individual members of supporting institutions.

Subscriptions, orders for numbers issued in the last three calendar years, and changes of address should be sent to Pacific Journal of Mathematics, P.O. Box 969, Carmel Valley, CA 93924, U.S.A. Old back numbers obtainable from Kraus Periodicals Co., Route 100, Millwood, NY 10546.

The Pacific Journal of Mathematics at P.O. Box 969, Carmel Valley, CA 93924 (ISSN 0030-8730) publishes 5 volumes per year. Application to mail at Second-class postage rates is pending at Carmel Valley, California, and additional mailing offices. Postmaster: send address changes to Pacific Journal of Mathematics, P.O. Box 969, Carmel Valley, CA 93924.

\section{PUBLISHED BY PACIFIC JOURNAL OF MATHEMATICS, A NON-PROFIT CORPORATION} Copyright (c) 1988 by Pacific Journal of Mathematics 


\section{Pacific Journal of Mathematics}

Vol. 134, No. $2 \quad$ June, 1988

P. D. Allenby and M. Sears, Extension of flows via discontinuous functions

Arthur William Apter and Moti Gitik, Some results on Specker's

problem

Shiu-Yuen Cheng and Johan Tysk, An index characterization of the catenoid and index bounds for minimal surfaces in $\mathbf{R}^{4}$

Mikihiro Hayashi and Mitsuru Nakai, Point separation by bounded analytic functions of a covering Riemann surface

Charles Philip Lanski, Differential identities, Lie ideals, and Posner's theorems

Erich Miersemann, Asymptotic expansion at a corner for the capillary problem

Dietrich W. Paul, Theory of bounded groups and their bounded cohomology

Ibrahim Salama, Topological entropy and recurrence of countable chains

Zbigniew Slodkowski, Pseudoconvex classes of functions. I. Pseudoconcave

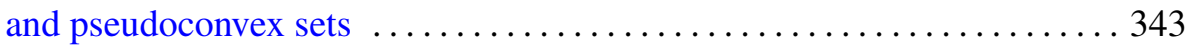

Alfons Van Daele, $K$-theory for graded Banach algebras. II . . . . . . . . . . 377

Edwin Wolf, Functions in $R^{2}(E)$ and points of the fine interior 393 